\title{
Further Explorations of Cellular Uptake of Radioactivity
}

I internal emitter therapy that radiopharmaceuticals are distributed nonuniformly within tissues of localization, and this nonuniformity extends to all spatial levels-organs, suborgans, multicellular clusters, single cells, and even subcellular compartments $(1,2)$. Furthermore, the dosimetric consequences of these distributions have been extensively studied, especially for shortrange radiations such as $\alpha$-particles and Auger electrons (3). Microdosimetric studies that investigate the biologic consequences of internal emitters have explicitly considered nonuniform radioactivity distributions, but in so doing, the

\section{See page 1009}

radioactivity per labeled cell was considered to be constant (i.e., equal to some mean value) $(4,5)$. Although such studies have given consideration to the fraction of labeled versus unlabeled cells in an organ, tissue, or a cell cluster, the paucity of experimental data on the distribution of activity per cell required the assumption of a constant value per labeled cell in these microdosimetric and cellular-level dosimetry studies.

In June 2006, Neti and Howell published an elegant experimental study that provided data on the distribution of activity per cell (6). Chinese

Received Mar. 5, 2008; revision accepted Mar. 6, 2008.

For correspondence or reprints contact: Wesley E. Bolch, University of Florida, 202 NSC, Gainesville, FL 32611-8300.

E-mail: wbolch@ufl.edu

COPYRIGHT (c) 2008 by the Society of Nuclear Medicine, Inc.

DOI: 10.2967/jnumed.108.050567 hamster V79 lung fibroblasts were exposed to varying concentrations of ${ }^{210} \mathrm{Po}$-citrate, and the activity per cell was measured via $\alpha$-particle autoradiography. Their studies thus gave direct experimental data that cellular uptake in cells is governed by a lognormal distribution and that the shape of that distribution remains unchanged with increases in the distribution mean. Their data was subsequently used to perform theoretic modeling of cell survival as a function of the mean activity per cell. The authors demonstrated substantial differences in cell survival shape when the activity per cell was lognormally distributed versus when it was assumed conventionally that every cell in the population contained the mean activity. This effect was particularly pronounced at cell survival fractions below $10 \%$.

In a subsequent Journal of Nuclear Medicine Letter to the Editor, Kvinnsland et al. (7) suggested that the statistical nature of radioactive decay can influence the measured distribution of $\alpha$-particle tracks, particularly at low numbers of tracks per cell as studied by Neti and Howell (6). In their response, the authors performed a statistical reanalysis of their original data to further elucidate whether or not Poisson statistics would alter their original conclusions. In an article in this issue of The Journal of Nuclear Medicine, Neti and Howell present an expansion of this reanalysis whereby 3 different distributions are reviewed (Poisson, lognormal, and Poisson-lognormal) to assess the distribution of $\alpha$-particle tracks per cell measured in their study (8). Their current study showed that at the lower activity concentrations, Poisson statistics do not significantly affect their original conclusions that distributions of lognormal activity concentra- tion are seen in cells exposed to ${ }^{210} \mathrm{Po}$-citrate, and that at their highest concentration, the Poisson-lognormal distribution perhaps better explains their data.

The statistical arguments put forth by these authors will be valuable to researchers working with cellular uptake models of radiopharmaceuticals as well as those looking at biologic response to internal emitters at the cellular level. Although these in vitro experiments are important sources of data for modeling radiobiologic response to therapy radiopharmaceuticals, the next significant challenge will be to acquire such data within experimental models of tissue structure, within tissues of laboratory animals, and eventually within treated patients in clinical trials. When possible, studies of radioactivity uptake should additionally, and when practical, simultaneously look at the distribution of target cells (if they are not always the same), as both are needed to fully understand biologic outcome (9). Whereas a multitude of factors can influence overall tissue response, ultimately the activity uptake within individual cells of the targeted tissue, as well as uptake within cells of the dose-limiting organ, must be understood to enable the construction of meaningful dose-response relationships, which in turn will eventually lead to improvements in radiopharmaceutical design and targeting strategy.

\section{Wesley E. Bolch \\ University of Florida, Gainesville, Florida}

\section{REFERENCES}

1. Howell RW, Wessels BW, Loevinger R. The MIRD perspective 1999. J Nucl Med. 1999;40(suppl): $3 \mathrm{~S}-10 \mathrm{~S}$.

2. International Commission on Radiation Units and Measurements (ICRU). Absorbed Dose Specification 
in Nuclear Medicine. Report 67. Bethesda, MD: ICRU; 2002.

3. Humm JL. Dosimetric aspects of radiolabeled antibodies for tumor therapy. J Nucl Med. 1986;27: 1490-1497.

4. Goddu SM, Rao DV, Howell RW. Multicellular dosimetry for micrometastases: dependence of self-dose versus cross-dose to cell nuclei on type and energy of radiation and subcellular distribution of radionuclides. $\mathrm{J} \mathrm{Nucl} \mathrm{Med.} \mathrm{1994;35:}$ $521-530$.
5. Faraggi M, Gardin I, Stievenart JL, Bok BD, Le Guludec D. Comparison of cellular and conventional dosimetry in assessing self-dose and cross-dose delivered to the cell nucleus by electron emissions of $99 \mathrm{mTC},{ }^{123} \mathrm{I},{ }^{111} \mathrm{In}$ ${ }^{67} \mathrm{Ga}$ and 201T1. Eur J Nucl Med. 1998;25 205-214.

6. Neti PV, Howell RW. Log normal distribution of cellular uptake of radioactivity: implications for biologic responses to radiopharmaceuticals. $\mathrm{J} \mathrm{Nucl}$ Med. 2006;47:1049-1058.
7. Kvinnsland Y, Stokke T, Aurlien E. Log normal distribution of cellular uptake of radioactivity [letter]. J Nucl Med. 2007;48:327.

8. Neti PVSV, Howell RW. Lognormal distribution of cellular uptake of radioactivity: statistical analysis of $\alpha$-particle track autoradiography. J Nucl Med. 2008;49:1009-1016.

9. Watchman CJ, Bourke VA, Lyon JR, et al. Spatial distribution of blood vessels and CD34+ hematopoietic stem and progenitor cells within the marrow cavities of human cancellous bone. J Nucl Med. 2007;48:645-654. 